Rapid Reviews COVID-19

\title{
Review 3: "Tocilizumab in Hospitalized Patients With COVID-19 Pneumonia"
}

Imran Khan ${ }^{1}$

${ }^{1}$ University of California Davis, Pathology \& Lab Medicine

Published on: Oct 16, 2020

DOI: 10.1162/2e3983f5.164c8373

License: Creative Commons Attribution 4.0 International License(CC-BY 4.0). 


\section{$\underline{\text { RR:C19 Evidence Scale rating by reviewer: }}$}

- Strong. The main study claims are very well-justified by the data and analytic methods used. There is little room for doubt that the study produced has very similar results and conclusions as compared with the hypothetical ideal study. The study's main claims should be considered conclusive and actionable without reservation.

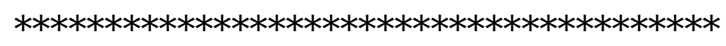

\section{Review:}

This is a well written research article on a critical topic, one of unusually high importance to biomedical research today. The authors have made a strong case for the benefit of Tocilizumab in the reduction of the number of days to hospital discharge/ready for discharge and duration of ICU. The study population is welldefined. This reviewer is not an expert in the evaluation of statistical analyses. The statistical methods used seem to be appropriate. However, for a more definitive evaluation, the editor may rely on their own judgement or another reviewer.

The following suggestions would improve the manuscript:

1. In Table 1, it would help to provide breakdown of 'Symptoms at diagnosis' by comorbidities (that are already listed). The added information would provide a better understanding of whether certain symptoms are more or less prevalent in certain comorbidities.

2. In Figure 2 (A \& B), under 'Patients remaining at risk' it would improve readability if $\%$ values of patients, in addition to the number provided, are also provided. 